\title{
Medical Compliance and Income-Health Gradients
}

\author{
By Anne Case, Ingrid le Roux, and Alicia Menendez**
}

Wealthier people live longer and experience less morbidity than do poorer people, in both developed and developing countries. While the association between income and health status has been well documented, the mechanisms leading to this correlation are unclear. In this paper, we use data collected from an informal urban township in South Africa to examine the extent to which compliance with medical protocols plays a role in the observed incomehealth gradient. Specifically, we look at adherence to protocols among individuals diagnosed with hypertension.

Hypertension is an interesting condition to focus on, for several reasons. Left untreated, it can lead to stroke, cardiovascular disease, and kidney failure. In one large demographic surveillance site in South Africa, cerebrovascular accidents were the second largest cause of death among adults aged 35-54 in the mid-1990's (Kathleen Kahn and Stephen M. Tollman, 1999). In principle, hypertension can be controlled, and blood pressure brought down into the normal range, through a combination of anti-hypertensive drugs, diet, and exercise (U.S. Department of Health and Human Services, 2003). In South Africa, Standard Guidelines and Essential Drug stepwise treatment for hypertension recommends monthly clinic visits, until a patient's blood pressure returns to the normal range, after which the patient is encouraged to be seen every three months (National Department of Health, 1998 p. 23). Our interest here is in analyzing whether household resources contribute to a respondent's ability or

\footnotetext{
* Case: Woodrow Wilson School of Public and International Affairs, Princeton University, Princeton, NJ 08544 (e-mail: accase@princeton.edu); le Roux: Philani Nutrition Center, 5 Glencoe Road, Oranjezicht, Cape Town, South Africa 8001 (e-mail: ile@princeton.edu); Menendez: Harris School, University of Chicago, 1155 E. 60th Street, Chicago, IL 60637 (e-mail: menendez@uchicago.edu). We gratefully acknowledge support from the National Institute on Aging (R01 AG20275-01) and expert research assistance from Shawna Samuel. We thank Angus Deaton for comments on an earlier draft.
}

willingness to take hypertensive medication. We find that, among the adults in our survey who know that they are hypertensive, those from poorer households are significantly less likely to be taking medication to control their blood pressure. Among hypertensive respondents in the highest income quartile, 47 percent are noncompliant, while among those in the lowest quartile, 75 percent report not currently taking medication for their condition.

\section{The Princeton Khayelitsha Survey (PKS) 2002}

Our data were collected from a random sample of 200 households surveyed between May and November 2002 in the first round of an ongoing data collection effort in Khayelitsha, a township of approximately 500,000 people on the outskirts of Cape Town. The township is composed of both houses that receive services (water and electricity) and unserviced shacks. Most households have a family connection to the Eastern Cape (Transkei, Ciskei), from which their families originally migrated. Unemployment and poverty rates in the township are extremely high, and the community faces major health problems in HIV/AIDS, TB, violence, and malnutrition.

For the PKS survey, a "knowledgeable household member" was first interviewed and asked questions about all persons in the household. Every adult identified as a household member was then interviewed separately and asked a battery of questions about physical and mental health, education, income, earnings, and expenditures. All were offered a follow-up free medical examination, performed by a physician, together with a chest X-ray, blood work (without HIV testing), and urine analysis. The PKS team volunteered to pick up all members of all households that participated in the survey and take them for laboratory tests and medical exams on a Saturday morning, and to return them to their homes at the end of their exams. 
TABle 1-Determinants of Participation in Medical Examinations

\begin{tabular}{lcc}
\hline \hline & \multicolumn{2}{c}{ Dependent variable } \\
\cline { 2 - 3 } & $\begin{array}{c}\text { Someone in } \\
\text { household } \\
\text { chose to } \\
\text { participate }\end{array}$ & $\begin{array}{c}\text { Respondent } \\
\text { chose to } \\
\text { participate }\end{array}$ \\
Controls [means] & no & yes \\
\hline Household fixed effects? & 0.178 & - \\
Log(household size) [1.46] & $(0.066)$ & \\
Indicator: household has & 0.118 & - \\
$\quad$ water on site [0.76] & $(0.088)$ & - \\
Log(household total income) & 0.021 & - \\
[7.14] & $(0.037)$ & - \\
Number luxury items owned & -0.060 & - \\
[1.37] & $(0.041)$ & 0.004 \\
Age [34.7] & - & $(0.001)$ \\
Female [0.53] & - & 0.111 \\
Holds a regular-pay job & & $(0.034)$ \\
[0.29] & & -0.070 \\
Reports health is fair or poor & - & $(0.041)$ \\
[0.38] & & 0.046 \\
Low education [0.09] & - & -0.152 \\
& & $(0.072)$ \\
No. observations: & 194 & 518 \\
\hline
\end{tabular}

Notes: OLS regressions are reported; numbers in parentheses are standard errors. $F$ test of the joint significance of water on site, $\log$ (household income), and the number of luxury items owned in column $1: F=0.96, p$ value $=0.4124$.

Knowledgeable household members named a total of 590 adults in the households interviewed. Of these, 525 (89 percent) agreed to complete an individual interview. For these individuals, we have self reports from 516 on whether a doctor, nurse, or health-care professional ever told them that they had a number of chronic conditions, including high blood pressure. Of the adults who participated in the PKS survey, 153 also completed a physical exam.

Survey respondents who chose to have a physical exam were not a random subset of all respondents and, in what follows, self-selection into medical exams should be borne in mind. Table 1 presents information on participation. Eighty-four households (42 percent) had at least one member take a medical exam. Column 1 examines participation at the household level, using an ordinary least-squares (OLS) regression of a participation indicator (equal to 1 if any member chose to participate) on several
TABLE 2-HyPERTENSION, PKS 2002

\begin{tabular}{lcc}
\hline \hline $\begin{array}{l}\text { Blood pressure category } \\
\text { [fraction of the sample] }\end{array}$ & $\begin{array}{c}\text { Systolic } \\
\text { reading }\end{array}$ & $\begin{array}{c}\text { Diastolic } \\
\text { reading }\end{array}$ \\
\hline Normal [30.3] & $<130$ & $<80$ \\
High normal [29.6] & $<140$ & $<90$ \\
Stage 1 hypertensive [24.3] & $<160$ & $<100$ \\
Stage 2 [8.6] & $<180$ & $<110$ \\
Stage 3 or higher [7.2] & $180+$ & $110+$ \\
\multicolumn{3}{c}{ (Number of observations $=152)$} \\
\hline
\end{tabular}

household-level variables. Larger households were more likely to have at least one member participate. However, we could find no other significant household-level correlate of participation. Household income, household ownership of "luxury" items (a car, a television, cable TV, a refrigerator, a personal computer), and having water on site are neither separately nor jointly significant predictors of household participation.

In column 2, we present determinants of individual respondents' decisions to participate in physical exams, from OLS regressions that control for household-level fixed effects. We find that, within households, participation is higher among older members and among women. Those with a "regular pay" job are less likely to come for a medical exam, perhaps because they are more likely to be covered by medical insurance through their work, or perhaps because they are busier on Saturdays than are members who are unemployed. Adults with less than two years of schooling are also less likely to come for a medical exam. Those members who selfreport their health as "fair" or "poor" are slightly more likely to participate, although the difference between members in poor health and other members is not statistically significant.

\section{Hypertension and Compliance}

Eighty-eight adults (17 percent) reported during their home-based interviews that a health-care professional had told them that their blood pressure was high. The quality of these self-reports can be assessed in part (and subject to possible selection bias) by seeing whether those who selfreport hypertension in their home-based interviews are more likely to be observed with high blood pressure during their medical exams. We categorize blood pressure as in Table 2. Respon- 
dents are Stage-3 hypertensive if either their systolic reading is above 180 , or their diastolic reading is above 110; Stage-2 hypertensive if either their systolic reading is between 160 and 180 , or their diastolic reading is between 100 and 110 , and they have not already been categorized as Stage 3; and so on. Forty percent of all adults who participated in our medical exams had high blood pressure, with 16 percent exhibiting Stage-2 hypertension or higher. This is consistent with the findings of other researchers that chronic diseases are a large problem for South Africans (Debbie Bradshaw et al., 2002), and that the need exists for better hypertension screening and treatment.

If all individuals were fully compliant in taking medication for high blood pressure, we would not expect to find self-reports to be predictive of observed hypertension. This is not the case: an ordered probit of blood pressure $(1=$ normal; $2=$ high normal; $3=$ Stage $1 ; 4=$ Stage 2; $5=$ Stage 3 or higher) on individuals' self-reports of high blood pressure yields a coefficient of 0.84 (standard error $=0.22$ ), demonstrating that self-reports are significantly correlated with the presence of hypertension. This may be because hypertensive people do not fully understand that medication must continue to be taken after blood pressure has been reduced. They may underestimate the risks they run if their hypertension remains unchecked, or they may find the time and money costs associated with getting to the clinic for treatment prohibitive.

Of the 88 adults who report having been told at some earlier point about their hypertension, only half (46 persons) reported that they were currently taking medication for this condition. An additional nine respondents reported that they were currently taking medication but were found in medical exams to be hypertensive at Stage 2 or higher. We will assume that respondents in this second group are either not taking their medications or are not taking them properly. The results presented below are similar if we either eliminate these nine respondents from our sample or treat them as compliant. In what follows, we will categorize the 42 adults who report not currently taking medication and the nine adults who report taking medication but were found to be dangerously hypertensive as "noncompliant."
TABle 3-Determinants of Noncompliance

\begin{tabular}{lc}
\hline \hline Variable [mean] & Coefficient \\
\hline Log(total household income) [mean = 7.44] & -0.157 \\
& $(0.068)$ \\
Log(household size) [1.67] & 0.280 \\
& $(0.150)$ \\
Educational attainment [6.01] & 0.013 \\
& $(0.023)$ \\
Depression index [1.14] & -0.009 \\
& $(0.032)$ \\
Indicator: regular pay job [0.42] & -0.033 \\
& $(0.120)$
\end{tabular}

No. observations:

86

Notes: The table reports probit regressions; numbers in parentheses are standard errors. The sample is restricted to adults who report knowing that they are hypertensive. The dependent variable $=1$ if respondent is known to be hypertensive but is not taking medication. Also included in the probit are an indicator that the respondent is female and the respondent's age. Coefficients reported here are changes in the probability of noncompliance, given a change in the right-side variable.

Information collected in the PKS survey allows us to examine several potential explanations for noncompliance. Recent work on insulin-dependent diabetes and on HIV antiretroviral therapy in the United States by Dana P. Goldman and James P. Smith (2002) suggests a causal role for education in compliance: better educated people may better understand the requirements of their therapies. (However, Goldman and Smith also note that, in their analysis of the Health and Retirement Survey, the addition of the Wechsler Adult Intelligence Score [WAIS] absorbs the effect of schooling on poor self-maintenance behaviors. They interpret this as evidence that the relationship between socioeconomic status and adherence to a medical regime may not be due to resource availability, but to individuals' abilities in higher-level reasoning.) We examine whether schooling plays a role in medicating for hypertension in the PKS survey and present results in Table 3. Controlling for age and an indicator that the respondent is female (not reported in the table), we find no predictive effect of education on compliance. This is true whether we control for years of completed schooling (as in Table 3 ) or add indicators that a respondent has little or no schooling (0 or 1 years) or is well educated (a high-school degree or greater). 
Moreover, the education of other household members (in particular, the maximum educational attainment of any member, and an indicator that some member has a high-school degree or greater) was not significantly associated with compliance. It may be that schooling was of such low quality for blacks in South Africa before the end of apartheid government that it is a very noisy marker for true years of schooling. In future research we hope to examine this further.

A second potential explanation involves depression. All adult respondents were asked whether, during the past month, "almost none of the time, some of the time, or most of the time," they felt miserable, sad, depressed, whether they cried a lot, did not feel like eating, found that their sleep was restless, "could not get going," and whether they felt everything they did was an effort. Three-quarters of all respondents said that they had experienced at least one of these "some of the time" during the past month, and over 30 percent reported that they had experienced at least one of these "most of the time." In Table 3, we test to see whether depression leads to a lack of compliance. We find little evidence for this explanation in our data. No marker for depression predicts lack of compliance with anti-hypertensive drugs. Table 3 presents the simple sum of the number of times individuals report that "most of the time" the statements being read were true for them during the past month. However, neither taking the markers of depression one-by-one, nor taking the markers based on a report that "some of the time" or "most of the time" respondents felt this way had any predictive effect for compliance.

A third explanation is that resource constraints stop people from being able to adhere to their protocols. We find that hypertensive respondents from households with greater total household income are significantly more likely to report that they are currently taking medication for high blood pressure. This can be seen in Table 3 where, controlling for age, sex, education, and depression, a 1-percent increase in total household income is seen to correspond to a 0.16-percentage-point increase in the probability of compliance. That this compliance is not due to the availability of private medical insurance is suggested by the fact that having a regular- pay job has no significant predictive power with respect to compliance.

\section{Discussion}

If all respondents faced equal risk of hypertension, and those from wealthier households were more likely to adhere to medical protocols after hypertension was diagnosed, then we would expect to find a negative relationship between observed hypertension and income. However, among those who participated in medical exams, we find no significant correlation between the two. While this could be due to self-selection into medical exams, it may also be due to the higher risk of hypertension faced by wealthier respondents in our survey. Obesity rises monotonically with income in our sample, and obese people are at higher risk of hypertension. Among adults who responded to our household-based questionnaire, self-reports of ever having been diagnosed as hypertensive increase with income. The lack of any significant relationship between observed hypertension and income may reflect differential compliance between richer and poorer respondents.

In 2004 we will return to the households we surveyed in 2002. We plan to ask a series of questions to better understand compliance. This will involve asking questions about the perceived risks that accompany hypertension, respondents' understandings of their need to continue anti-hypertensive drugs after their blood pressure returns to a normal range, and the costs they face in adhering to their treatments.

\section{REFERENCES}

Bradshaw, Debbie; Schneider, Michelle; Dorrington, Rob; Bourne, David E. and Laubscher, Ria. "South African Cause-of-Death Profile in Transition." South African Medical Journal, August 2002, 92(8), pp. 618-23.

Goldman, Dana P. and Smith, James P. "Can Patient Self-Management Help Explain the SES Health Gradient?" Proceedings of the National Academy of Science, August 2002, 99(16), pp. 10929-34. 
Kahn, Kathleen and Tollman, Stephen M. "Stroke in Rural South Africa." South African Medical Journal, January 1999, 89(1), pp. 63-65.

National Department of Health, Government of South Africa. Standard treatment guidelines and essential drug list for South Africa, primary health care, 1998 Ed. Pretoria, South Africa: National Department of Health, 1998.
[Available online: 〈www.doh.gov.za/docs/ factsheets/ $/$.]

U.S. Department of Health and Human Services. The seventh annual report of the Joint $\mathrm{Na}$ tional Committee on Prevention, Detection, Evaluation, and Treatment of High Blood Pressure, NIH Publication No. 03-5233. Washington, DC: National Heart, Lung, and Blood Institute, May 2003. 